\title{
Analysis of Toxicity of Ceramic Nanoparticles and Functional Nanocomposites Based on Vulcanized Natural Rubber
}

\author{
Felipe Silva Bellucci ${ }^{1,2, a}$; Jeferson Camargo Fukushima ${ }^{1}$; \\ Josué de Moraes ${ }^{3}$; Marcos Augusto Lima Nobre ${ }^{4}$; \\ Amarildo Tabone Paschoalini ${ }^{\Upsilon}$; Aldo Eloizo Job ${ }^{4}$ \\ ${ }^{1}$ School of Natural Sciences and Engineering - UNESP, 15385-000, Ilha Solteira, Brazil \\ ${ }^{2}$ Ministry of Science Technology and Innovation - MCTI, CEP 70067-900, Brasilia, DF, Brazil \\ ${ }^{3}$ Research Center for Neglected Diseases - NPDN, Guarurulhos, SP, Brazil \\ ${ }^{4}$ School of Technology and Applied Sciences - UNESP, 19060-900, Presidente Prudente, SP, Brazil
}

\begin{abstract}
Nanocomposites are multiphase materials of which, at least one of the phases, has a dimension smaller than $100 \mathrm{~nm}$. These materials have attracted technological and scientific interest due to their multifunctional characteristics and potential, which allow them to combine unique properties which are not found in traditional commercial materials, such as natural rubber alone. The objective of this work is to analyse the toxicity of nanoparticles and nanocomposites when applied to mammal cells in order to obtain bioactive agents, as well as to evaluate the potential to be applied in biological systems. Ferroelectric ceramic nanoparticles of KSr2Nb5O15 (KSN) and paramagnetic ceramic nanoparticles $\mathrm{Ni} 0.5 \mathrm{Zn} 0.5 \mathrm{Fe} 2 \mathrm{O} 4$ (NZF) were prepared and utilized to produce functional and multifunctional nanocomposites based on vulcanized natural rubber (NR/KSN and NR/NZF) with different nanoparticle concentrations. For both kinds of nanoparticles and both classes of nanocomposites, independently of the nanoparticle concentration, it is not possible to observe any reduction of the cellular viability until the incubation time is finished. In this way, these results point to the possibility of using these nanoparticles and nanocomposites, from the toxicity point of view, as bioactivity agents in biological systems based on mammalian cells.
\end{abstract}

\section{Introduction}

The nanocomposite field is related to the study of multiphase materials, of which at least one of the phases has a dimension smaller than $100 \mathrm{~nm}$. Nanocomposites are promising materials as there are ranges of possible nanoparticles combinations to obtain a specific material with intended properties, which are not found in traditional ones. Nowadays, studies have been carried out to improve the control of the dispersion of nanometric components on polymeric matrices, and the understanding of the interactions between structures which are chemically diverse [1]. The use of nanoparticles with paramagnetic, ferromagnetic, piezoelectric, pyroelectric or ferroelectric properties in a matrix, such as polymeric matrix, allows the nanocomposite to have differentiated characteristics from the nanoparticles used. Some electrical applications can be restricted due to the heat caused by the dielectric loss, when electrical fields with high magnitude are present, or in some specific frequencies when alternating electric fields are applied. Moreover, magnetic nanocomposites, whose magnetic particles are dispersed either in magnetic or non-magnetic matrix, present many technological applications, 3 of which can be mentioned here: magnetic

\footnotetext{
${ }^{\mathrm{a}}$ Corresponding author: bellucci@dem.feis.unesp.br
}

recording, giant magnetoresistance (GMR) and magnetic refrigeration. [2].

Neglected tropical diseases (NTDs) are a diverse group of diseases which go beyond poverty conditions, contributing to the perpetuation of economic and social inequality. The following diseases can be mentioned as examples of neglected diseases: Leishmaniosis, dengue, Chagas disease, schistosomiasis, leprosy, malaria, tuberculosis, among others [3]. Consequently, multidisciplinary research involving materials and biotechnology areas have been gaining attention in order to develop new materials and methods, which can be used to combat such diseases. In the field of biotechnology, natural rubber together with its composites, emerge as potential candidates for a new generation of bioactive agents with biocidal characteristics. This is because natural rubber and its nanocomposites are able to stimulate angiogenic processes, and its presents a good capacity to disperse particulate loads [4]. However, studies combining the fields of nanometric materials and public health have a poor record in literature, therefore, more research is needed in this area.

The main objective of this paper is to analyse the toxicity of ferroelectric $\mathrm{KSr}_{2} \mathrm{Nb}_{5} \mathrm{O}_{15}$ and paramagnetic $\mathrm{Ni}_{0.5} \mathrm{Zn}_{0.5} \mathrm{Fe}_{2} \mathrm{O}_{4}$ nanoparticles and functional and multifunctional nanocomposites based on vulcanized 
natural rubber on mammal cells. This kind of analysis is the first step towards using these materials in biological systems.

\section{Materials and methods}

\subsection{Synthesis of phases KSr2Nb5015 and Ni0.5Zn0.5Fe2O4 via Modified Polyol Method}

The nickel-zinc ferrite is a typical polycation oxide exhibiting a spinel type structure with a complex cation distribution in the crystalline structure. The unitary cell has been represented by the formula $\left(\mathrm{Zn}^{2+}{ }_{\mathrm{x}} \mathrm{Fe}^{3+}{ }_{1-\mathrm{x}}\right)\left[\mathrm{Ni}^{2+}{ }_{\mathrm{x}}\right.$
$\left.\mathrm{Fe}^{3+}{ }_{1+\mathrm{x}}\right] \mathrm{O}^{2-}{ }_{4}$, where, $\mathrm{Zn}$ cations occupies only tetrahedral sites, Ni cations occupy only octahedral sites, while Fe cations occupy both tetrahedral and octahedral sites. Potassium strontium niobate with stoichiometry $\mathrm{KSr}_{2} \mathrm{Nb}_{5} \mathrm{O}_{15} \quad(\mathrm{KSN})$, is a unipolar ferroelectric with spontaneous polarization only along the crystallographic [001] direction and a high dielectric constant. The reagents applied to the synthesis of the oxides KSN and NZF by using the MPM [5] are highlighted in Table 1. The materials obtained for both phases showed an average crystalline size smaller than $15 \mathrm{~nm}$ and details of the synthesis process can be obtained in $[5,6]$.

Table 1. Chemical compounds used for the synthesis of particles $\mathrm{KSr}_{2} \mathrm{Nb}_{5} \mathrm{O}_{15}$ and $\mathrm{Ni}_{0.5} \mathrm{Zn}_{0.5} \mathrm{Fe}_{2} \mathrm{O}_{4}$.

\begin{tabular}{|c|c|c|c|c|}
\hline Chemical compounds & Chemical formula & Purity & Molecular Mass & Origin \\
\hline \multicolumn{5}{|c|}{ Nanoparticles of $\mathrm{KSr}_{2} \mathrm{Nb}_{5} \mathrm{O}_{15}$} \\
\hline Strontium Carbonate & $\mathrm{SrCO}_{3}$ & A. P.* & $147.6 \mathrm{~g} / \mathrm{mol}$ & VETEC \\
\hline Potassium Carbonate & $\mathrm{K}_{2} \mathrm{CO}_{3}$ & A. P. & $138.2 \mathrm{~g} / \mathrm{mol}$ & VETEC \\
\hline Niobium Salt & $\mathrm{NH}_{4} \mathrm{H}_{2}\left[\mathrm{NbO}\left(\mathrm{C}_{2} \mathrm{O}_{4}\right)_{3}\right] \cdot 3 \mathrm{H}_{2} \mathrm{O}$ & A. P. & $477.8 \mathrm{~g} / \mathrm{mol}$ & CBMM \\
\hline \multicolumn{5}{|c|}{$\begin{array}{ll}\text { Nanoparticles of } \mathrm{Ni}_{0.5} \mathrm{Zn}_{0.5} \mathrm{Fe}_{2} \mathrm{O}_{4} \\
\end{array}$} \\
\hline Nickel Oxide & $\mathrm{Ni}_{2} \mathrm{O}_{3}$ & A. P. & $165.4 \mathrm{~g} / \mathrm{mol}$ & NUCLEAR \\
\hline Zinc Oxide & $\mathrm{ZnO}$ & A. P. & $129.4 \mathrm{~g} / \mathrm{mol}$ & VETEC \\
\hline Iron Oxide & $\mathrm{Fe}_{2} \mathrm{O}_{3}$ & A. P. & $111.7 \mathrm{~g} / \mathrm{mol}$ & VETEC \\
\hline
\end{tabular}

* Analytical Purity (A.P.)

\subsection{Preparation of the ferroelectric and paramagnetic nanocomposites.}

The preparation of the ferroelectric composites $\mathrm{NR} / \mathrm{KSN}$ and paramagnetic composites NR/NZF, started with the mixture of the activating system, plus KSN or NZF nanoparticles using different concentrations of 1,3 , $5,10,20$ and $50 \mathrm{phr}$, together with $100 \mathrm{phr}$ of dried natural rubber type "crepe claro brasileiro", by using an opened chamber mixer for over 20 minutes. The mixture which was produced was kept at room temperature and with no exposure to light for 24 hours. After this step, the acceleration and vulcanization system based on sulphur $\left(\mathrm{S}_{8}\right)$ was added to the activated sample in the same way. Moreover, these accelerated samples were then thermoconformed to membranes of $1.5 \mathrm{~mm}$, and thin films of $200 \mu \mathrm{m}$ thickness, by using a heated-plate press at $150^{\circ} \mathrm{C}$, as this temperature is indicated for the vulcanization process of natural rubber [6]. As a result of the sample preparation mentioned previously, the following samples were obtained: NR, NR/KSN$1,3,5,10,20$ and 50phr, and NR/NZF-1,3,5,10,20 and 50phr. As expected, a satisfactory volumetric homogeneity was obtained for all samples investigated indicating that the preparation method, system and vulcanization parameters used were appropriate. The dispersion, interaction and size effect of nanometer-sized particles in the polymer matrix, in special rubber matrices, is reported to have a significant impact on the mechanical properties of composites and nanocomposites.

\subsection{Cell viability and toxicity tests}

The cell viability and toxicity tests of nanoparticles, vulcanized natural rubber and nanocomposites on mammal cells were conducted by using the Violet Crystal Method, as described by J. Moraes and his collaborators [7]. The Vero ATCC CCL-81 were the mammal cell lines used in this work. Such cells are from "American Type Culture Collection" (Manassas, VA, USA), which is a kidney cell line of African green monkeys Cercopithecus aethiops (L.). For the experiments, the Vero cells were cultivated on culture plates with 96 boreholes and different concentrations of nanoparticles between 15.6 and $1000 \mu \mathrm{g} / \mathrm{mL}$, or by using nanocomposites of vulcanized natural rubber matrix in concentrations between 250 and $4000 \mu \mathrm{g} / \mathrm{mL}$ in DMEM (Dulbecco's Modified Eagle Medium), which was supplemented with $10 \%$ of fetal bovine serum (FBS) at $37^{\circ} \mathrm{C}$, in an atmosphere of $\mathrm{CO}_{2}$ at $5 \%$. These composites were in contact with mammal cells and, after 24 and 48 hours, the supernatant portion was removed and, then the agglutinated cells were fixed and coloured by using violet crystal $0.2 \%$ in methanol $20 \% \mathrm{v}$. The toxicity was evaluated via the absorbance of control boreholes containing cells in DMEM medium. Moreover, the cultures were monitored daily during the whole incubation period by using an inverted optical microscope. All the tests were carried out three times and the calculated mean standard deviation was smaller than $2 \%$. 


\section{Results and discussions}

Nowadays, there is a need for innovation in the biotechnological field, so nanoparticles and nanocomposites are potentially candidates for a new generation of biocide agents. Moreover, tests that evaluate the toxicity of such materials on mammal cells are a very important step in the process of biotechnology development. Figure 1 shows the results related to the assessment of toxicity (or viability) of Vero cells after a 48 hour incubation period, in the presence of ceramic nanoparticles $\mathrm{KSr}_{2} \mathrm{Nb}_{5} \mathrm{O}_{15}$ (a) and $\mathrm{Ni}_{0.5} \mathrm{Zn}_{0.5} \mathrm{Fe}_{2} \mathrm{O}_{4}$ (b), together with their respective constituent elements as a function of particle concentration in the cell medium.
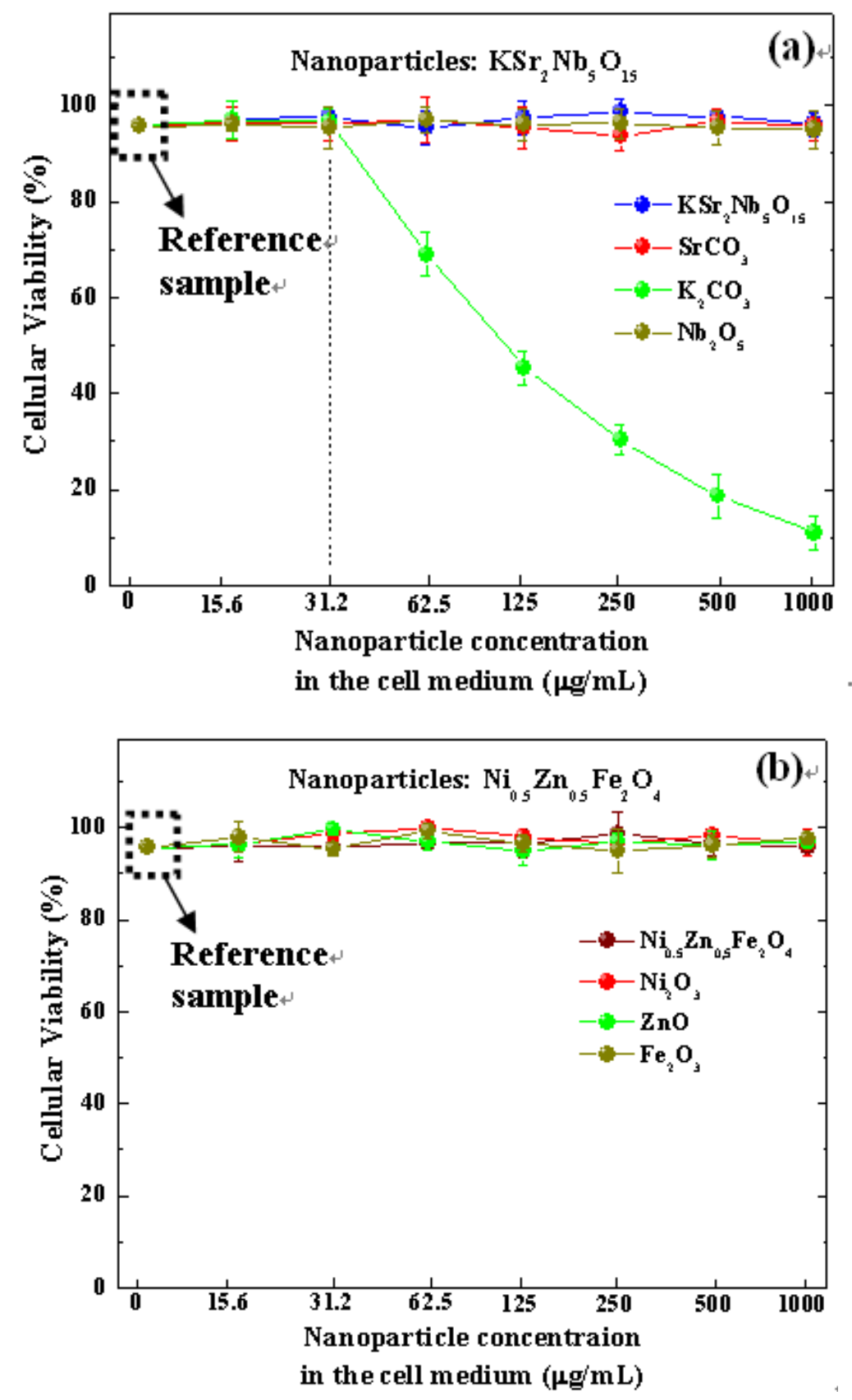

Figure 1. Cell viability test in the presence of ferroelectric nanoparticles (a), magnetics (b), and their respective constituent elements as a function of particles dispersed in the culture medium. Mammal cells (Vero type) were used in this test, and were cultivated in the presence of particles

As observed in Fig. 1, there is no statistically significant reduction in the cell viability after finishing the incubation period, over the concentrations used in these tests, where the maximum concentration is 1000 $\mu \mathrm{g} / \mathrm{mL}$ when compared to incubated cells in culture medium. Although this is the case for the majority of the two ceramic phases and their constituent elements, the potassium carbonate $\mathrm{K}_{2} \mathrm{CO}_{3}$ is an exception. In this case, a statistically significant reduction $(\mathrm{P}<0.01)$ of the cell viability for concentrations higher than a $62.5 \mu \mathrm{g} / \mathrm{mL}$ 
inclusive is clearly observed. This is because $\mathrm{K}_{2} \mathrm{CO}_{3}$, in an aqueous medium, tends to dissociate into potassium ions $\left(\mathrm{K}^{+}\right)$transforming the extracellular medium, which should be hypotonic, into a highly hypertonic medium. Hence, the cells undergo a heavy water loss process through the cytoplasmic membrane, leading to an imbalance in essential mechanisms for cellular maintenance, such as, the sodium/potassium pump, mechanisms responsible for conducting nervous impulses, protein synthesis and cellular respiration. The combination of these processes is the more likely reason for the death of mammal cells when exposed to particles of $\mathrm{K}_{2} \mathrm{CO}_{3}$. However, for the ferroelectric phase $\mathrm{KSr}_{2} \mathrm{Nb}_{5} \mathrm{O}_{15}$, the potassium ions $\left(\mathrm{K}^{+}\right)$are isolated in the pentagonal sites of the crystallographic structure, which avoid the presence of such ions in the extracellular medium. Hence, reduction of cells when nanoparticles of $\mathrm{KSr}_{2} \mathrm{Nb}_{5} \mathrm{O}_{15}$ are used is not observed.

Figure 2 shows the result of the toxicity (or viability) test of Vero cells after 48 hours of incubation, in the presence of vulcanized natural rubber, ferroelectric nanocomposites NR/KSN (a) and magnetics (b) as a function of the concentration of nanocomposite. Moreover, pictures of cells exposed to NR/KSN-50phr and NR/NZF-50phr, together with the reference sample, are also shown. These pictures were collected by using optical microscopy.

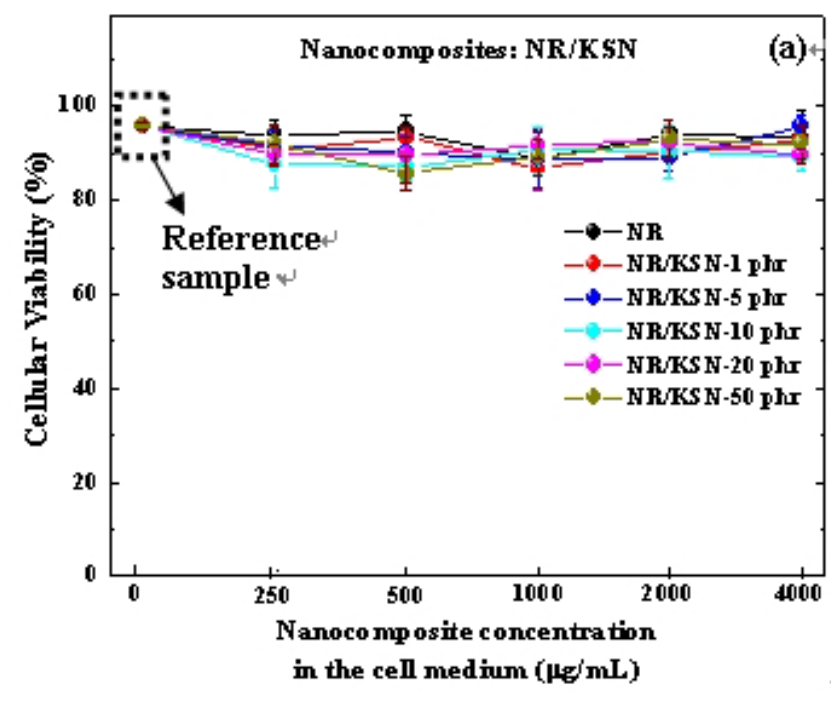

NR/KSN-50 phr
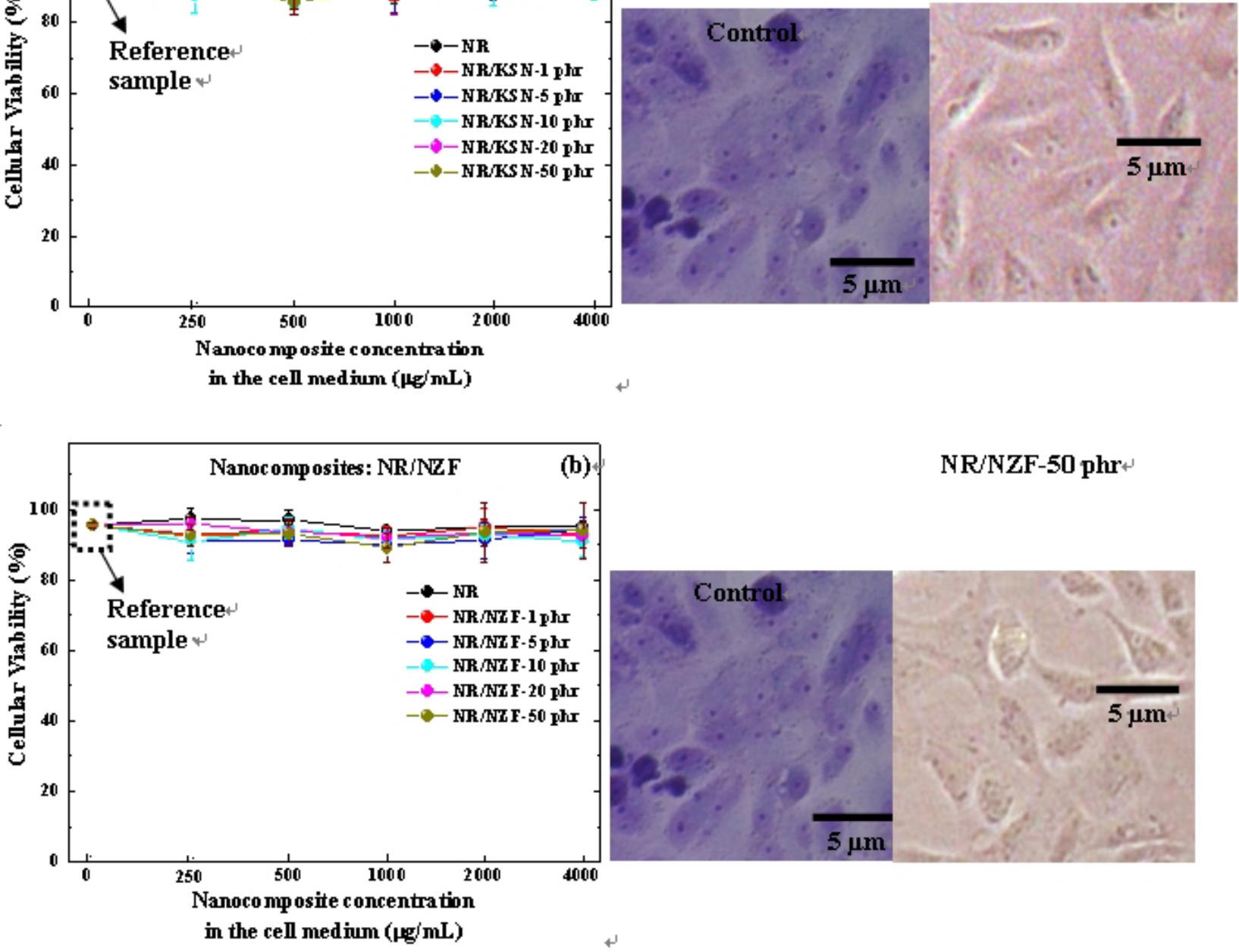

NR/NZF-50 phr

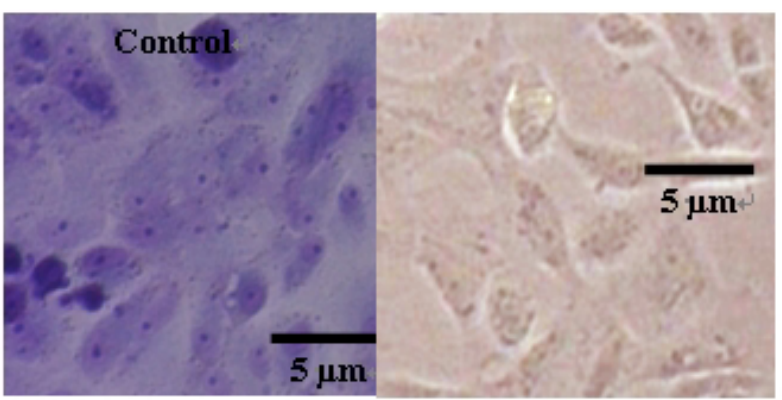

Figure 2. Cellular viability of the culture in the presence of ferroelectric nanocomposites NR/KSN (a) and magnetic nanocomposites $\mathrm{NR} / \mathrm{NZF}(\mathrm{b})$ as a function of the sample concentration $(\mu \mathrm{g} / \mathrm{mL})$ in the cellular medium. The pictures show the cells exposed and not exposed to the nanocomposites.

It can be observed in Fig. 2 that for vulcanized natural rubber, and for both nanocomposites, no matter the concentration of nanoparticles, there is no statistically significant reduction of the cell viability after the incubation period is finished. It is observed that in both pictures generated by using optical microscopy, there are adhered cells to the substrate, suggesting that the cells are still viable (feasible). Moreover, no significant morphological change in the cells exposed to the two types of nanocomposites is observed, and the one in the 
medium by itself (control/reference sample), suggesting that the mammal cells were not greatly affected by the presence of nanocomposites. Hence, these systems are able to be used in biological systems composed of mammals cells, as there is no significant reduction in terms of cellular viability when such cells are exposed to nanoparticles of $\mathrm{KSr}_{2} \mathrm{Nb}_{5} \mathrm{O}_{15}, \mathrm{Ni}_{0.5} \mathrm{Zn}_{0.5} \mathrm{Fe}_{2} \mathrm{O}_{4}$, vulcanized natural rubber and nanocomposites. This finding has led to new tests, which evaluate the bioactive and biocide effects of these materials, therefore providing encouraging results. It can be mentioned that ferroelectric and paramagnetic nanocomposites have been used as modulation agents to develop colonies of Leishmaniose brasiliense and schistomiasis, as already tested using natural rubber nanocomposites with silver nanoparticles [8].

\section{Conclusions}

In this paper the modified polyol method (MPM) was used in the chemical synthesis of ferroelectric oxide potassium strontium niobate with $\mathrm{KSr}_{2} \mathrm{Nb}_{5} \mathrm{O}_{15}$ stoichiometry and of the paramagnetic oxide nickel-zinc ferrite with $\mathrm{Ni}_{0.5} \mathrm{Zn}_{0.5} \mathrm{Fe}_{2} \mathrm{O}_{4}$ stoichiometry. Moreover, monophasic particles of an average crystalline size on the nanometric scale were obtained. A method based on dry mixture and solid state reaction was employed to prepare the functional and multifunctional nanocomposites with different concentrations of nanoparticles. Toxicity tests were conducted, and no dramatic changes were observed for cellular viability when mammal cells were exposed to nanoparticles of $\mathrm{KSr}_{2} \mathrm{Nb}_{5} \mathrm{O}_{15}, \mathrm{Ni}_{0.5} \mathrm{Zn}_{0.5} \mathrm{Fe}_{2} \mathrm{O}_{4}$, vulcanized natural rubber and nanocomposites. Hence, it seems that these systems, which were mentioned previously, are able to be used in biological systems composed of mammal cells. Furthermore, this result suggests that these materials can be also used as bioactive agents in biological systems, as well as modulation agents for the development of colonies of different types of parasites.
The authors acknowledge the Brazilian research agencies FAPESP ( $\left.n^{\circ} 2009 / 00523-8\right)$, CAPES $\left(n^{\circ}\right.$ BEX $5129 / 09-5$ and $\left.n^{\circ} 11208 / 13-9\right), C N P q\left(n^{\circ} 480377 / 2013-8\right.$, 455323/2014-3, 454843/2014-3 and 449999/2014-9) and FEPISA ( $\left.\mathrm{n}^{\circ} 010 / 2014\right)$ for their financial support. The main author is a post-doctorate researcher in FEISUNESP.

\section{References}

1. F. S. Bellucci, E. R. Budemberg, M. A. L.Nobre, J. A. De Saja, R. F. Aroca, M. A. Rodríguez-Pérez and A. E. Job: Sci. Adv. Mater, 5(6), (2013), p.637-646.

2. V. Sunny, P. Kurian, P. Mohanan, P.A. Joy and M.R. Anantharaman, J. Alloys and Comp., 489, (2010), p. 297.

3. Department of Science and Technology, Secretariat of Science, Technology and Strategic Inputs, Ministry of Health. Neglected diseases: Strategies of the Ministry of Health. Rev. Saúde Pública, 44, (2010), p. 200.

4. M. Ferreira, R. J. Mendonça, J. Coutinho-Netto and M. Mulato, Braz. J. Phys., 39, (2009), p. 564.

5. F. S. Bellucci: Dielectric characterization of nanometric and nanostructured particles of niobate oxide from tetragonal tungsten bronze family with stoichiometry KSr2Nb5O15. 2009. 156 f. Master Tesis-Universidade Estadual Paulista.

6. F. S. Bellucci, Preparation and characterization of multifunctional nanocomposites obtained with ferroelectric and paramagnetic nanoparticles in natural rubber films. 2013. 185 f. Ph.D. TesisUniversidade Estadual Paulista.

7. J. Moraes, C. Nascimento, P. O. Lopes, E. Nakano, L. F. Yamaguchi, M. J. Kato and T. Kawano, Exp. Parasitol., 127, (2011), p. 357.

8. C. G. Barboza-Filho, F.C. Cabrera, R.J. Dos Santos, J.A. De Saja Saez and A.E. Job. Exp Parasitol., 130, 2, (2012), p.152-158.

\section{Acknowledgements}

